\title{
Iatrogenic Oesophageal Perforations in Neoplastic Lesions: Management with Covered Self-Expanding Prostheses
}

\author{
Mario Anselmi Mendez ${ }^{1}$, Ana MaríA Gemmato Pascazio', \\ Maximiliano Figueroa Silva², Julio Salgado OyarzÚN² \\ ${ }^{1}$ Surgery Department, School of Medicine, University of Concepción, Concepción, Chile \\ ${ }^{2}$ Endoscopic Surgery Diploma Program Fellow, Department of Surgery, School of Medicine, University of Concepción, \\ Concepción, Chile \\ Email: manselmi@udec.cl
}

How to cite this paper: Mendez, M.A., Pascazio, A.M.G., Silva, M.F. and OyarzÚN, J.S. (2021) Iatrogenic Oesophageal Perforations in Neoplastic Lesions: Management with Covered Self-Expanding Prostheses. Open Journal of Gastroenterology, 11, 89-104.

https://doi.org/10.4236/ojgas.2021.116010

Received: May 3, 2021

Accepted: June 27, 2021

Published: June 30, 2021

Copyright $\odot 2021$ by author(s) and Scientific Research Publishing Inc. This work is licensed under the Creative Commons Attribution International License (CC BY 4.0).

http://creativecommons.org/licenses/by/4.0/

\begin{abstract}
Background: Perforation of the oesophagus is a serious condition. Most of them are iatrogenic and are associated with significant morbidity and mortality, especially with late diagnosis. Aim: To prospectively analyse the results of the endoscopic management of iatrogenic perforations in oesophageal neoplasia, through the immediate insertion of a covered self-expanding prosthesis (CSES). Materials and Methods: Between 01.01.2006 and 12.30.2016, a series of 19 consecutive patients attended the Teaching Unit of Endoscopic Surgery of the Regional de Concepción Hospital, Chile, with the diagnosis of oesophageal neoplasia confirmed by biopsy were prospectively studied. All were subjected to a prior evaluation by the oncology team and subsequently referred for endoscopic palliative management of dysphagia. The average age was $77 \pm 9.3$ years, $8(42.1 \%)$ were female and $11(57.9 \%)$ were male. In 17 patients $(89.5 \%)$ the stenosis compromised the oesophagus, in $2(10.5 \%)$ the gastro-oesophageal junction, 16 had a squamous carcinoma (84.2\%) and 3 an adenocarcinoma (15.8\%). Results: Perforation was diagnosed during the procedure in 18 patients (94.7\%) and in 1 (5.3\%) 22 hours later. Follow-up was done for a minimum of 90 days or until death. The prostheses were inserted successfully in all cases. The immediate evolution was satisfactory in 12 patients (63.2\%). In the rest (36.8\%), 18 complications appeared. The most frequent were retrosternal pain, subcutaneous emphysema and fever. The early evolution was satisfactory in $12 / 19$ cases (63.2\%). Complication was evidenced in 7 (36.8\%), the most frequent was retrosternal pain $(36.8 \%)$. Fever occurred in $3(15.8 \%)$, pleural effusion in $3(15.8 \%)$ and mediastinitis in 2 of these. The prosthesis was kept in situ as a definitive palliation method for
\end{abstract}


neoplastic dysphagia. In 10 of the 18 cases that survived more than a month, there were late complications (55.6\%), none of them associated with the perforation itself. The only death (5.3\%) was due to an oesophagus-pleural fistula, associated with an early prosthetic migration. Recovery of the oral intake occurred, on average, at 3.7 days. The hospital stay averaged 9.6 days. Conclusion: The use of CSES for the treatment of iatrogenic oesophageal perforations in the context of neoplasia, is a safe and effective method, with low morbidity, adequate recovery of the oral intake and prompt discharge from hospital.

\section{Keywords}

Iatrogenic Oesophageal Perforations, Self-Expanding Prostheses, Oesophageal Cancer

\section{Introduction}

Perforation of the oesophagus remains one of the most feared adverse events of diagnostic and therapeutic endoscopy. Iatrogenic injuries represent up to $60 \%$ of all cases [1]. However, these are not frequent; their incidence fluctuates between 3.1 to $4.7 / 1,000,000$ per year [2] [3].

Endoscopic dilation of the oesophagus is a long-established technique for treating benign strictures of this organ [4]. It has also been used to manage dysphagia in unresectable malignant lesions as a sole palliative measure [5] or as a step before endoprosthesis implantation [6].

The main complication of oesophageal dilation is perforation; this is associated with morbidity that can reach up to $40 \%$ and a mortality of $27 \%$ [7] [8] [9]. Advances in endoscopic equipment have improved the safety of the procedure [10]. However, complications still occur, even in the most experienced hands.

For most of the $20^{\text {th }}$ century, surgical therapy was considered the norm in managing acute perforation of the oesophagus. Although surgery continues to play an important role, in the last decade, treatment has evolved towards a significant reduction in its use, with an increase in non-surgical procedures such as interventional endoscopic and radiology [11].

The use of endoluminal therapies to treat oesophageal leaks and perforations has grown exponentially over the last decade. In the right circumstances, it offers many advantages compared to traditional surgical intervention [12].

In recent years, new therapeutic endoscopic techniques have been developed to treat oesophageal perforation and reduce the morbidity and mortality associated with it; these include clips, sutures, self-expanding covered metal prostheses (CSES), and endoluminal vacuum therapy [12].

Stents appear to be the best option in perforations that occur in the context of a malignant lesion because clips and sutures tend to tear the neoplastic tissue and do not hold the edges of the perforation [13].

Oesophageal prostheses, initially limited to palliation of dysphagia in malig- 
nant neoplasia [6] [14], are currently being used successfully to treat, among others, anastomotic leaks [15], benign strictures [16], spontaneous perforations [17], tracheoesophageal fistulas [18], and oesophageal variceal haemorrhage [19].

The time interval between the perforation and the start of treatment is one of the most important factors affecting the therapeutic outcome. Indeed, most authors suggest that when there is a delay in treatment greater than $24 \mathrm{~h}$., mortality doubles or triples [20] [21] [22] [23].

The ideal treatment for iatrogenic oesophageal perforation would minimize the negative impact of the treatment while sealing the organ's continuity solution as early as possible.

This study aims to prospectively analyse the results of the endoscopic management of iatrogenic oesophageal neoplastic perforations through the immediate insertion of a covered self-expanding prosthesis.

\section{Patients and Method}

Between 01 January 2006 and 31 December 2016, a series of 19 consecutive patients attended the Teaching Unit of Endoscopic Surgery of the Regional de Concepción Hospital, Chile, with the diagnosis of oesophageal neoplasia confirmed by biopsy. All were subjected to a prior evaluation by the oncology team and referred for endoscopic palliative management of dysphagia.

\section{INCLUSION CRITERIA}

- Obstructive neoplasm of the oesophagus;

- Referred to endoscopic palliation from oncology;

- Severe dysphagia;

- Iatrogenic oesophageal lesion;

- Accepted endoscopic management of iatrogenic complication.

The study was conducted following the ethical standards of Good Medical Practice following current ethical standards (Declaration of Helsinki).

The mean age of the group was $77 \pm 9.3$ years (range 67 - 93 years); eight patients $(42.1 \%)$ were female and $11(57.9 \%)$ were male. In 13 patients $(68.4 \%)$, the stenosis compromised the middle oesophagus, in four $(20.1 \%)$ the distal, and in two (10.5\%) the gastro-oesophageal junction. The types of neoplastic lesion corresponded to squamous carcinoma in 16 patients (84.2\%) and adenocarcinoma in three (15.8\%).

Of the 19 cases studied, 14 (73.7\%) attended dilation for the first time, and five (26.3\%) had previously been dilated. In eight of them (42.1\%), dilation was indicated as a palliative treatment for dysphagia, and in the remaining 11 (57.9\%) as a step before the insertion of a self-expanding metal prosthesis. All patients were dilated using Savary-Guillard bougies (Wilson-Cook Medical Inc. Winston-Salem, N.C). In the former, an attempt was made to obtain the largest possible oesophageal lumen in the initial session [5], while in the latter, it was only dilated to $38 \mathrm{~F}[6]$.

The length of the lesion was determined by radiology and endoscopy. 
Seven patients (35\%) had a history of having undergone radiotherapy and presented post-radiation tumour recurrence, which in turn was the cause of dysphagia.

In 18 of the 19 patients (94.7\%), the diagnosis of perforation was made in the same operative act or immediately afterwards during the routine endoscopic and fluoroscopic review established in the protocol for this type of procedure. In the remaining patient (5.3\%), to whom a CSES was inserted as palliation of cardio-oesophagal neoplasia, the diagnosis was made $22 \mathrm{~h}$ after the procedure, when he consulted with a clinical picture suggestive of mediastinitis, which was confirmed by a thoracic Computed Tomography (CT).

The procedures were carried out under strict protocols established by our centre, including radiological control with a water-soluble contrast medium (Hypaque $^{\circledR}$ ) before and after the procedure.

All the interventions were performed by previously obtaining the informed consent of each patient or her legal representative.

Video endoscopes [models TJF 145-150-160-180 (Olympus Optical Co., Tokyo, Japan)] were used for the dilations and the insertion of the CSES.

Once the complication was established, a self-expanding prosthesis was inserted. All stents were placed in the same operative act or immediately after diagnostic confirmation. The insertion technique was described in a previous study [6].

The dilations and insertion of prostheses were performed with the patient in the left lateral decubitus and under control of their vital signs using a multi-parameter monitor.

Before the intervention, the patients underwent topical pharyngeal anaesthesia with $2 \%$ Lidocaine gel or $4 \%$ spray. Of the total, 17 patients (89.5\%) received intravenous sedation with Midazolam $(0.5-5 \mathrm{mg})$ and eventual analgesia with Meperidine $(10-30 \mathrm{mg})$, as is pre-established in the unit's protocol. In the remaining two cases $(10.5 \%)$, the intervention was performed with anaesthetics support due to their comorbidities.

All procedures were performed by an endoscopic surgeon or endoscopic surgery residents, under the strict supervision of the former.

Prostheses of various types, lengths, diameters, and coverage were used according to the extent of the tumour lesion and perforation, location, and availability of the resource at the time of trauma (Table 1).

Table 1. Types of self-expanding prosthesis and their coverage $(n=19)$.

\begin{tabular}{ccc}
\hline Type of prosthesis & Type of Partial & Coverage Total \\
\hline Choostent & 4 & 6 \\
Hannarostent & 2 & 2 \\
Ultraflex & 1 & 2 \\
Poliflex & & \\
Evolution & 2 & \\
\hline
\end{tabular}


In patients treated between 01 January 2006 and 30 June $2012(\mathrm{n}=13), 18$ $\mathrm{mm}$. diameter prostheses were used, fully covered in ten patients $(76.9 \%)$ and partially covered in the remaining three patients (23.1\%). From 07 January 2012 to 30 December $2016(\mathrm{n}=6)$, only $22 \mathrm{~mm}$ diameter prostheses were used; the majority were partially covered (5/6).

After the stent was deployed, the leaks of the contrast medium were ruled out by carrying out a new oesophageal transit, then starting endoscopic and fluoroscopic follow-ups at 24 and $48 \mathrm{~h}, 7$ days, and then once per month, as stipulated in the protocols of our unit. If a leak was found, then the sealing process, using a covered stent, started immediately (Figures 1-4).

Once the prosthesis was inserted, the patients were hospitalized. Zero regimens were indicated for 24 to $48 \mathrm{~h}$. Intravenous antibiotics, serial clinical, laboratory and imaging controls, hospital visits, or daily communication with patients

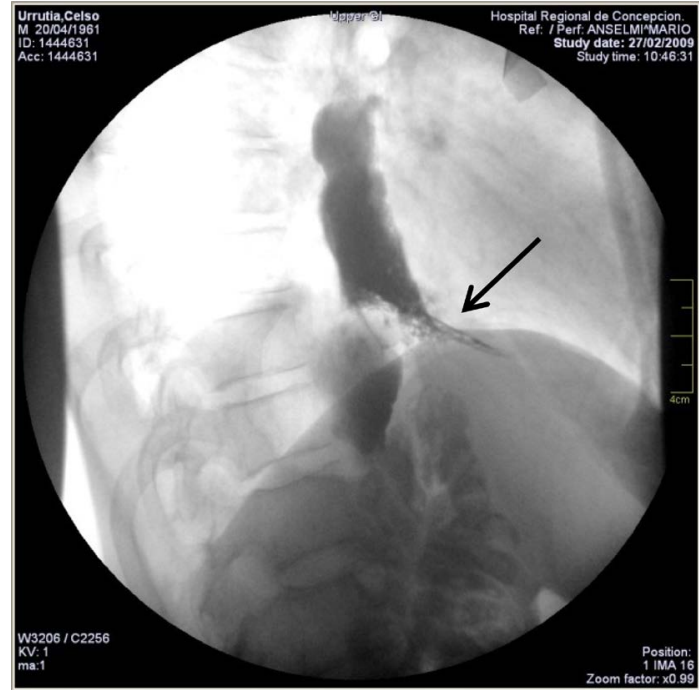

Figure 1. Radiological control with a water-soluble contrast medium (Hypaque ${ }^{\circledR}$ ) swallow after bougie dilation procedure. The arrow shows contrast leakage in a patient with iatrogenic perforation of the oesophagus.

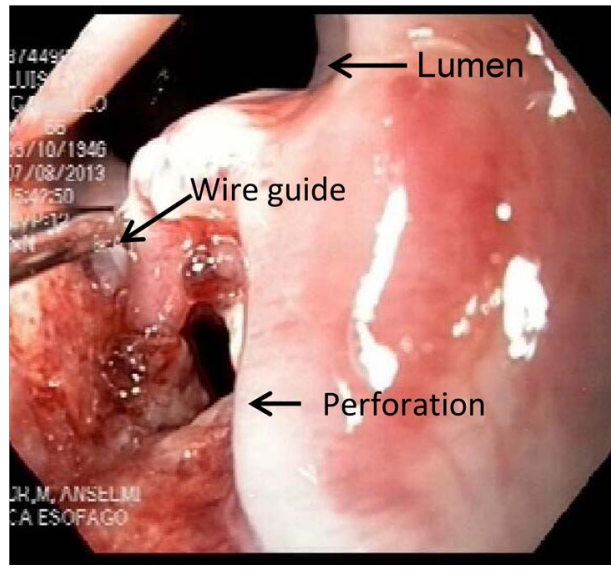

Figure 2. Endoscopic view of an iatrogenic oesophageal neoplasm perforation immediately after dilation. 


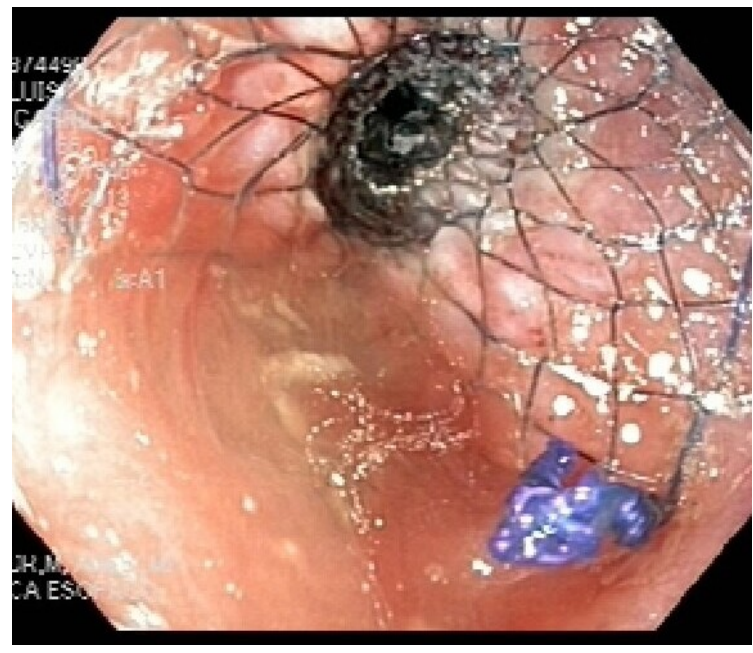

Figure 3. Endoscopic view of the covered stent sealing of the perforation. Visible residue of contrast medium in the proximal end of the stent.

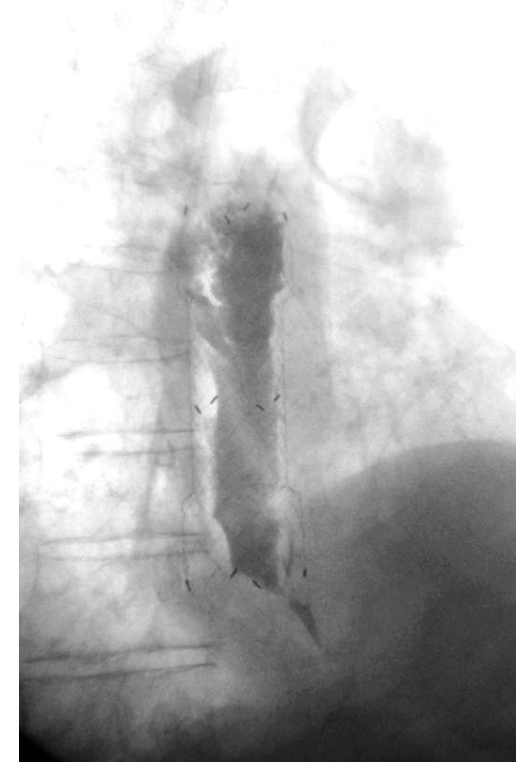

Figure 4. Radiological control with a water soluble contrast medium (Hypaque ${ }^{\circledR}$ ) after inserting the covered stent. No leakage is observed.

or their treating physicians in search of complications secondary to iatrogenic injury or stent insertion was carried out.

The appearance of symptoms associated with the perforation event was analysed. Immediate complications were considered those that appeared within the first $24 \mathrm{~h}$. after the perforation was established: early, those diagnosed in the first 30 days; and late, those that occurred 30 or more days after the stent was installed.

Follow-up was done for a minimum of 90 days or until the death of the patient. The recovery of the ability to feed orally, plus the absence of complications derived from the perforation that required subsequent surgical management or associated with mortality, was considered successful in managing iatrogenic 
complication.

\section{Results}

A total of 19 patients were studied. The prostheses were inserted successfully in all cases. This was done during the same procedure in which the perforation occurred in $19 / 19$ patients (100\%). In one patient (5.3\%), severe desaturation occurred during insertion, which had to be suspended momentarily. This patient was in poor general condition, for which anaesthetic support was requested, and the anaesthesiologist successfully managed the complication. The insertion of the prosthesis was carried out without problems.

The immediate evolution $(<24 \mathrm{~h})$ was satisfactory in 12 of the 19 patients studied (63.2\%). In the seven patients in whom immediate morbidity appeared (36.8\%), there were 18 complications (2.6 per patient). The most frequent were retrosternal pain $(\mathrm{n}=7 ; 36.8 \%)$, subcutaneous emphysema $(\mathrm{n}=3 ; 15.8 \%)$, and fever $(\mathrm{n}=3 ; 15.8 \%)$. One patient, who was dilated to $38 \mathrm{Fr}$. as a step before the insertion of the prosthesis, presented fever and retrosternal pain, verifying $22 \mathrm{~h}$ later the appearance of a pneumomediastinum. In a patient, the presence of air in the peritoneal cavity was demonstrated by $\mathrm{CT}$, which had no consequences. The cumulative frequency of immediate complications is shown in Table 2.

The early evolution was satisfactory in $12 / 19$ cases (63.2\%). Morbidity was evidenced in $7 / 19$ patients (36.8\%), the most frequent being retrosternal pain (36.8\%). This disappeared after $48 \mathrm{~h}$. in five of the seven cases presenting it, and before the tenth day in the remaining two. The rest of the early complications and their cumulative frequency are listed in Table 3.

In three patients $(15.8 \%)$, there was the persistence of fever, and in three (15.8\%), a pleural effusion appeared, in two of whom drainage using an endopleural tube was required. Furthermore, in two of these cases (10.5\%), mediastinitis was confirmed. One presented symptoms suggestive of sepsis with dyspnea, tachycardia, pain, and an effusion secondary to pneumonia. This corresponded to the only case in which the perforation went unnoticed during dilation (38 Fr.) before the insertion of the prosthesis, which was successful. Then, $22 \mathrm{~h}$. later, a chest CT scan was requested due to the symptoms, which confirmed the

Table 2. Immediate evolution $(<24 \mathrm{~h}$.)

\begin{tabular}{ccc}
\hline Morbidity & $\mathrm{n}=7 / 19$ & $\%$ \\
\hline Satisfactory & 12 & 63.2 \\
Retrosternal pain & 7 & 36.8 \\
Subcutaneous emphysema & 3 & 15.8 \\
Fever & 3 & 15.8 \\
Pneumothorax & 2 & 10.5 \\
Pneumomediastinum & 2 & 10 \\
Pneumoperitoneum & 1 & 5 \\
\hline
\end{tabular}


Table 3. Early evolution.

\begin{tabular}{ccc}
\hline Morbidity type & $\mathbf{n}=7 / 19$ & $\%$ \\
\hline Satisfactory & 12 & 63.2 \\
Retrosternal pain & 7 & 36.8 \\
Fever & 3 & 15.8 \\
Pleural effusion & 3 & 15.8 \\
Subcutaneous emphysema & 2 & 10.5 \\
Mediastinitis & 2 & 10.5 \\
Pneumonia & 1 & 5.3 \\
Pleural oesophagus fistula & 1 & 5.3 \\
Prosthesis migration & 1 & 5.3 \\
\hline
\end{tabular}

clinical suspicion of mediastinitis, probably secondary to the procedure. This patient responded satisfactorily to medical management, with hospital discharge 21 days later.

The second case of mediastinitis was associated with CSES migration 17 days after insertion and corresponded to an 84-year-old patient with oesophagal cancer treated with radiotherapy. A local recurrence was found, proven by upper gastrointestinal endoscopy and biopsy. The patient was referred for endoscopic palliative management of dysphagia using dilation with bougies. Two dilations were made, up to Fr. 51 and 57 respectively, both without incident. One month later and, due to recurrence of dysphagia, a new dilation was necessary. Due to the patient's comorbidities, the procedure was performed under anaesthetic support, dilating up to $57 \mathrm{Fr}$. Two hours later, the anaesthetic effect ended. According to the work protocol, an oesophageal transit was performed that allowed observing a contrast leak at the level of the distal oesophagus, establishing the diagnosis of instrumental perforation of the oesophagus.

A fully covered Choostent-type CSES measuring $110 \mathrm{~cm}$. in length and 18 $\mathrm{mm}$. in diameter was immediately installed. During the same procedure, a thoracoabdominal X-ray was performed, observing signs suggestive of a right pneumothorax, for which an endopleural tube was installed, achieving complete lung expansion.

The usual follow-up was carried out at 24 and $48 \mathrm{~h}$, checking the proper position and functioning of the CSES, with no evidence of leakage. After an airtightness test with oral methylene blue, it was decided to start feeding by mouth on the fifth day.

Seventeen days after the insertion of the prosthesis, there is an abrupt change in the evolution of the patient, who had been until that moment satisfactory. There was a significant increase in the debt due to the endopleura tube and the appearance of clinical and laboratory signs compatible with sepsis. The presence of a pleural oesophageal fistula was suspected, secondary to the migration of the prosthesis. This complication was confirmed with a radiological control and 
then with the administration of methylene blue orally, observing its exit by the pleura tube.

The patient quickly worsened, and given her condition, she was considered outside the therapeutic scope, maintaining only supportive treatment. The patient died 19 days after the procedure, constituting the only cause of mortality in this series (5.3\%).

Once the acute condition secondary to perforation had been overcome, the prosthesis was kept in situ in all patients as a definitive palliation method for dysphagia of neoplastic origin.

Of the 18 cases that survived more than 1 month, 10 (55.6\%) had late complications, none of them associated with the perforation itself (Table 4 and Table 5).

There was a late reappearance of dysphagia in six patients (33.3\%); this was due in two cases to fracture of the prosthesis and joint epithelial hyperplasia. It was necessary to insert a second stent (86 and 133 days after perforation). In two patients, this was secondary to epithelial hyperplasia at the proximal end of the device, which was resolved in both cases by dilation with bougies.

In one case, an impacted foreign body was found at $34 \mathrm{~cm}$. of the dental arch, which was removed endoscopically and in the remainder, the prosthesis migrated to the stomach. This last case was a patient with severe dysphagia and undergoing radiotherapy treatment, who had been inserted a Polyflex removable plastic prosthesis measuring $21 \mathrm{~mm}$. in diameter by 90 in length to allow adequate intake for the therapy duration and its removal according to the result of this. Once the migration was verified, the patient was dilated to $54 \mathrm{Fr}$. The prosthesis was removed without incident. Given the patient's conditions and the absence of dysphagia, he was kept under observation. One month later, he presented a foreign body in the oesophagus that pushed into the stomach and dilated again to $54 \mathrm{Fr}$. The patient remained without dysphagia until his death.

Table 4. Late evolution.

\begin{tabular}{ccc}
\hline & $\mathbf{n}=18$ & $\%$ \\
\hline Satisfactory & 9 & 50 \\
Dysphagia recurrence & 6 & 33.3 \\
Migration & 2 & 11.1 \\
Foreign body & 1 & 5.6 \\
Gastroesophageal reflux & 1 & 5.6 \\
\hline
\end{tabular}

Table 5. Causes of recurrent dysphagia.

\begin{tabular}{ccc}
\hline & $\mathbf{n}=6$ & $\%$ \\
\hline Prosthesis fracture & 2 & 33.3 \\
Epithelial hyperplasia & 2 & 33.3 \\
Foreign body & 1 & 16.7 \\
Prosthesis migration & 1 & 16.7 \\
\hline
\end{tabular}


A patient with a lesion in the distal oesophagus due to the insertion of a transcardial prosthesis without a valve presented gastro-oesophageal reflux whose symptoms resolved with the usual measures.

The average time elapsed between the perforation and the recovery of oral intake was 3.7 days, ranging between 1 to 8 days. In three cases (15.7\%), it was restarted in the first $24 \mathrm{~h}$. after inserting the prosthesis.

The hospital stay averaged 9.6 days, with a range between 2 and 21 days. The longest were those of the two patients who developed mediastinitis, one of who corresponds to the case of prosthetic migration and who died on the 19th day of hospitalization.

Patients survived an average of $252 \pm 188$ days (range 59-814 days). At the end of the follow-up, all 18 patients who survived had regained their ability to feed themselves orally and did not require additional surgical treatment to that described.

Statistical Analysis:

Descriptive statistics and percentage relative frequency were used.

\section{Discussion}

Oesophageal perforation is a life-threatening entity that requires early diagnosis and treatment [24]. These points are especially important in iatrogenic perforations secondary to endoscopic dilations. In these, complications are unpredictable, despite knowledge of risk factors, such as neoplastic and actinic stenosis and strict adherence to protocols in the safe application of procedures [25] [26].

The time interval between the perforation and the start of treatment is one of the most important factors affecting the therapeutic result. It has been suggested that early diagnosis and treatment, within the first $24 \mathrm{~h}$. after perforation is established, is the only way to avoid sepsis and ultimately minimize mortality. Indeed, most authors suggest that mortality doubles or triples when there is a delay in treatment greater than $24 \mathrm{~h} \mathrm{[20]} \mathrm{[21]} \mathrm{[22]} \mathrm{[23]} \mathrm{[24].}$

Due to the lack of a protective serous surface, perforations in the oesophagus can easily expand into the surrounding tissues and cause greater systemic complications derived from mediastinal and pleural contamination [1].

To make an early diagnosis, a high index of suspicion [27] and a strict follow-up protocol of patients are required, especially if it is a group with a higher risk of perforation, such as dilations of an obstructive neoplastic lesion. Indeed, in this group of patients, immediately after each therapeutic procedure, a dynamic radiological study with water-soluble contrast medium and/or endoscopy was performed in search of extraluminal leaks or mucosal lesions suspicious for perforation.

In this way, immediate detection of the lesion was achieved in 18/19 patients (94.7\%). In contrast, in the remaining patient (5.3\%), the diagnosis was made 22 $\mathrm{h}$ after the complication was established and was confirmed by CT thorax. This patient had been sent for the palliation of severe dysphagia through the insertion 
of a prosthesis. It only dilated to $38 \mathrm{Fr}$., and a fully covered self-expanding metal stent was inserted without incident. However, a few hours later, he presented symptoms suggestive of sepsis with dyspnea, tachycardia, and retrosternal pain. The patient had already sealed the perforation with the prosthesis, which was found to be properly located. The patient responded satisfactorily to medical management, leaving the hospital 21 days later.

In this series, the early detection rate $(<24 \mathrm{~h})$ was $100 \%$, different from that of others, in which figures of up to $63 \%$ are reported [27].

Fortunately, iatrogenic oesophageal perforation is a rare complication. Concerning the total number of patients, the incidence found was $0.03 \%$, while concerning the total dilations, it was $0.018 \%$ (Table 6 and Table 7). These figures are within limits published in the world literature, which oscillate between 0.02 and $1.5 \%$ [2] [3].

In recent years, the aetiology and management of oesophageal perforations have changed. The classic treatment techniques and protocols based on complex and lengthy surgical procedures, with high complication rates, have been replaced, in the right circumstances, by less invasive and safer conservative techniques with lower associated morbidity rates [24] [27]. Thus, new and revolutionary endoscopic devices have appeared for these purposes, including different types of clips, sealants, endoscopic sutures, and plastic and metal self-expanding

Table 6. Iatrogenic perforations in Oesophageal-Cardial Neoplasms in 12 years. Endoscopic Surgery Unit. Department of Surgery. University of Concepción. Chile.

\begin{tabular}{ccccccc}
\hline Year & Esophago & Cardias & Patients & Dilation & Dilat/pat & Perforations/year \\
\hline 2006 & 47 & 6 & 53 & 127 & 2.4 & 1 \\
2007 & 36 & 1 & 37 & 68 & 1.8 & 1 \\
2008 & 59 & 11 & 70 & 127 & 1.8 & 2 \\
2009 & 40 & 15 & 55 & 168 & 3.1 & 2 \\
2010 & 45 & 4 & 49 & 121 & 2.5 & 1 \\
2011 & 53 & 9 & 62 & 149 & 2.4 & 3 \\
2012 & 30 & 5 & 35 & 45 & 1.3 & 3 \\
2013 & 34 & 29 & 55 & 63 & 1.1 & 1 \\
2014 & 38 & 34 & 57 & 72 & 1.3 & 2 \\
2015 & 47 & 37 & 84 & 110 & 1.3 & 1 \\
2016 & 24 & 17 & 31 & 41 & 1.3 & 2 \\
Total & 453 & 168 & 588 & 1091 & 1.85 & 19 \\
\hline
\end{tabular}

Table 7. Oesophageal perforation according to the number of patients and dilations.

\begin{tabular}{ccc}
\hline & $\mathbf{n}$ & $\%$ \\
\hline Perforation per patient & $19 / 588$ & 0.03 \\
Perforation per dilation & $19 / 1091$ & 0.017 \\
\hline
\end{tabular}


prostheses [11] [12].

There is also an important advance in the perioperative management of patients, in which the appearance of new broad-spectrum antibiotics, the technological advances associated with intensive care units, and the optimization of nutritional support, play a fundamental role in the course of this complication [28] [29] [30].

In general, the surgical management of oesophageal perforations presents a morbidity of up to $75 \%$ [23] [27] [31] [32], and an associated mortality of $44 \%$ [23] [32] [33] [34].

On the other hand, conservative management in selected patients, is associated with a mortality close to $28.6 \%$ [1] [29] [35] [32], with complications that require subsequent surgical management in the order of $20 \%$ [29]. These figures clearly differ from those obtained in this study, in that the mortality associated with the complication occurred in one patient (5.3\%), while the need for surgical treatment was limited to the insertion of a chest tube for the management of a pleural effusion in 2 cases.

Early recognition and timely treatment are the most important factors influencing the outcome [20] [24]. In the patients studied, the diagnosis was made before 24 hours in $100 \%$ of the cases. In $18 / 19$ patients (94.7\%) during the procedure itself and in the remaining case (5.3\%) 22 hours after it. This allowed for the immediate insertion of a covered prosthesis, which quickly sealed the perforation, preventing significant mediastinal contamination. These factors undoubtedly positively influenced the good results obtained with the endoscopic management of this complication.

In this series, various types of prostheses were used for the treatment of perforation, depending mainly on the availability of the resource at the time of the adverse event and the characteristics of the injury. In the first 13 cases, $18 \mathrm{~mm}$. diameter prostheses were inserted, fully covered in 10 (76.9\%) and partially covered in the remaining $3(23.1 \%)$. However, since the appearance of the only case of prosthetic migration, which secondarily generated an oesophago-thoracic fistula, empyema, sepsis and finally the only case of mortality, it was deemed appropriate to change the type of CSES to be used in the new cases. Thus, subsequently, only partially covered $22 \mathrm{~mm}$. diameter prostheses were used. The objective of the above was to improve the anchoring of the CSES to the esophageal mucosa, both due to the greater diameter of the prosthetic opening, as well as the possibility of tissue growth through the uncovered framework of the ends of the device.

Considering migration, as the main complication inherent to the insertion of the prosthesis, it is noteworthy that in this series the percentage of this event was low, reaching 5.3\% compared to the average $15 \%$ found in the literature [34] [36]. The foregoing, probably due to the fact that all but one had severe stenosis secondary to the tumour.

The different endoscopic methods for the management of esophageal perfora- 
tions have shown advantages in reducing morbidity and mortality in selected patients [37]. The previous point becomes especially important in the group of patients considered in this study, in which due to the underlying stenosing neoplastic lesion, the prosthesis is considered as an alternative treatment, both for the management of the acute complication, in this perforation, as for palliation of dysphagia [36] [38].

The prostheses were inserted successfully in all cases. During the procedure two patients had problems. One, a severe desaturation, which merited the momentary suspension of the event without preventing it. The other underwent dilation only up to $38 \mathrm{Fr}$. and then the insertion of a prosthesis, the perforation going unnoticed, which manifested as mediastinitis, making the diagnosis 22 hours later. Both patients evolved satisfactorily.

The most frequent immediate complication was retrosternal pain, which in most cases subsided after 48 hours, as is the case usually in neoplastic strictures [6]. Subcutaneous emphysema, pneumomediastinum, and pneumoperitoneum were gradually reduced until they disappeared. The two cases that presented pneumothorax had to be drained using an endopleural tube, with satisfactory subsequent evolution. After 30 days, the prostheses behave as expected in a device designed to alleviate dysphagia, presenting the same late complications, all of which can be resolved endoscopically [6].

In relation to the economic and practical aspects associated with this complication, an important factor is the hospital stay, which in our series averaged 9.6 days, very similar to that of other publications, in which an average of 10.5 days are reported [36] [37].

Despite this data concordance, regarding this point, we consider that these patients could be discharged earlier and in many cases managed on an outpatient basis, with strict controls in the specialized referral units. In fact, 10 of the 19 patients in this study and after the insertion of the CSES, were transferred to low complexity hospital centres close to their homes for their initial clinical monitoring. These were re-sent to our referral unit to be controlled clinically, radiologically or endoscopically, as established in the protocols for this type of procedure, a limitation of this study is the number of patients, however this is an infrequent disease worldwide. Therefore, so far there are no randomized studies that have compared the results of the different therapeutic options and the recommended therapies vary, rather according to personal preferences or experiences than to the results of controlled studies.

From the results of this study, it can be concluded that the use of CSES for the treatment of iatrogenic oesophageal perforations, in the context of neoplasia, is a safe and effective method, with low morbidity, adequate recovery of the oral intake and prompt discharge from hospital.

\section{Conflicts of Interest}

The authors declare no conflicts of interest regarding the publication of this pa- 
per.

\section{References}

[1] Brinster, C.J., Singhal, S., Lee, L., Marshall, M.B., Kaiser, L.R. and Kucharczuk, J.C. (2004) Evolving Options in the Management of Esophageal Perforation. The Annals of Thoracic Surgery, 77, 1475-1483.

https://doi.org/10.1016/j.athoracsur.2003.08.037

[2] Vidarsdottir, H., Blondal, S., Alfredsson, H., Geirsson, A. and Gudbjartsson, T. (2010) Oesophageal Perforation in Iceland a Whole Population Study of Incidence, Aetiology and Surgical Outcome. The Thoracic and Cardiovascular Surgeon, 58, 476-480. https://doi.org/10.1055/s-0030-1250347

[3] Søreide, J.A., Konradsson, A., Sandvik, O.M., Øvrebø, K. and Viste, A. (2012) Esophageal Perforation: Clinical Patterns and Outcomes from a Patient Cohort of Western Norway. Digestive Surgery, 29, 494-502. https://doi.org/10.1159/000346479

[4] Anselmi, M. (1993) Estenosis benigna del esófago: Dilatación mediante el método de Celestin. Revista Chilena de Cirugia, 45, 326-331.

[5] Anselmi, M. and Silva, I. (1995) Endoscopic Dilation as a Method of Palliation of Malignant Strictures of the Esophagus and the Cardia. Revista Chilena de Cirugia, 47, 524-529.

[6] Anselmi, M., Gemmato, A.M. and Rodríoguez, A. (2012) Use of Choostent Prosthesis Device for Palliation of Dysphagia Secondary to Oesophageal Cancer. Revista Chilena de Cirugía, 64, 546-554. https://doi.org/10.4067/S0718-40262012000600008

[7] Zimmermann, M., Hoffmann, M., Jungbluth, T., Bruch, H.P., Keck, T. and Schloericke, E. (2016) Predictors of Morbidity and Mortality in Esophageal Perforation: Retrospective Study of 80 Patients. Scandinavian Journal of Surgery, 106, 126-132.

[8] Muir, A.D., White, J., McGuigan, J.A., et al. (2003) Treatment and Outcomes of Oesophageal Perforation in a Tertiary Referral Centre. European Journal of Cardio-Thoracic Surgery, 23, 799-804. https://doi.org/10.1016/S1010-7940(03)00050-2

[9] Okten, I., Cangir, A.K., Ozdemir, N., Kavukcu, S., Akay, H. and Yavuzer, S. (2001) Management of Oesophageal Perforation. Surgery Today, 31, 36-39. https://doi.org/10.1007/s005950170217

[10] Standards of Practice Commitee, Egan, J.V., Baron, T.H., et al. (2016) Esophageal Dilation. Gastrointestinal Endoscopy, 63, 755-760.

[11] Carrot Jr., P.H. and Low, D.E. (2011) Advances in the Management of Esophageal Perforation. Thoracic Surgery Clinics, 21, 541-555. https://doi.org/10.1016/j.thorsurg.2011.08.002

[12] Watkins, J.R. and Farivar, A.S. (2018) Endoluminal Therapies for Esophageal Perforations and Leaks. Thoracic Surgery Clinics, 28, 541-554. https://doi.org/10.1016/j.thorsurg.2018.07.002

[13] Raju, G.S. (2009) Endoscopc Closure of Gastrointestinal Leak. American Journal of Gastroenterology, 104, 1315-1320. https://doi.org/10.1038/ajg.2009.34

[14] Saranovic, D., Djuric-Stefanovic, A., Ivanovic, A., Masulovic, D. and Pesko, P. (2005) Fluoroscopically Guided Insertion of Self-Expandable Metal Esophageal Stents for Palliative Treatment of Patients with Malignant Stenosis of Esophagus and Cardia: Comparison of Uncovered and Covered Stent Types. Diseases of the Esophagus, 18, 230-238. https://doi.org/10.1111/j.1442-2050.2005.00484.x 
[15] Dai, Y., Chopra, S., Kneif, S. and Hünerbein, M. (2011) Management of Esophageal Anastomotic Leaks, Perforations and Fistulae with Self Expanding Plastic Stents. Journal of Thoracic and Cardiovascular Surgery, 141, 1213-1217. https://doi.org/10.1016/j.jtcvs.2010.07.096

[16] Ham, Y.H. and Kim, G.H. (2014) Plastic and Biodegradable Stents for Complex and Refractory Benign Esophageal Strictures. Clinical Endoscopy, 47, 295-300. https://doi.org/10.5946/ce.2014.47.4.295

[17] Dumonceau, J.-M. and Deviere, J. (2000) Treatment of Boerhaaave's Syndrome Using the Ultraflex Self-Expandable Stent. Gastrointestinal Endoscopy, 51, 773-774. https://doi.org/10.1016/S0016-5107(00)70117-9

[18] Hürtgen, M. and Herber, S.C. (2014) Treatment of Malignant Tracheoesophageal Fistula. Thoracic Surgery Clinics, 24, 117-127. https://doi.org/10.1016/j.thorsurg.2013.09.006

[19] Holster, I.L., Kuipers, E.J., van Buuren, H.R., Spaander, M.C. and Tjwa, E.T. (2013) Self-Expandable Metal Stents as Definitive Treatment for Esophageal Variceal Bleeding. Endoscopy, 45, 485-488. https://doi.org/10.1055/s-0032-1326227

[20] Rodríguez A, Braghetto I, Csendes A, Díaz Jc, Korn O, Burdiles P, et al. (2004) Current Results of the Management of Oesophageal Perforation. Revista Chilena de Cirugía, 56, 539-544.

[21] Hasimoto, C.N., Cataneo, C., Eldib, R., et al. (2013) Efficacy If Surgical versus Conservative Treatment in Esophageal Perforation: A Systematic Review of Case Series Studies. Acta Cirúrgica Brasileira, 28, 266-271. https://doi.org/10.1590/S0102-86502013000400006

[22] Biancari, F., Saarnio, J., Mennander, A., Hypén, L., Salminen, P., Kuttila, K., et al. (2014) Outcome of Patients with Esophageal Perforations: A Multicenter Study. World Journal of Surgery, 38, 902-909. https://doi.org/10.1007/s00268-013-2312-2

[23] Schmidt, S.C., Strauch, S., Rosch, T., et al. (2010) Management of Esophageal peRforations. Surgical Endoscopy, 24, 2809-2813. https://doi.org/10.1007/s00464-010-1054-6

[24] Kiev, J., Amendola, M., Bouhaidar, D., Sandhu, B.S., Zhao, X. and Maher, J. (2007) A Management Algorithm for Esophageal Perforation. The American Journal of Surgery, 194, 103-106. https://doi.org/10.1016/j.amjsurg.2006.07.024

[25] Piotet, E., Escher, A. and Monnier, P. (2008) Esophageal and Pharyngeal Strictures: Report on 1,862 Endoscopic Dilatations Using the Savary-Gilliard Technique. European Archives of Oto-Rhino-Laryngology, 265, 357-364. https://doi.org/10.1007/s00405-007-0456-0

[26] Tuna, Y., Koçak, E., Dinçer, D. and Köklü, S. (2012) Factors Affecting the Success of Endoscopic Bougia Dilatation of Radiation-Induced Esophageal Stricture. Digestive Diseases and Sciences, 57, 424-428. https://doi.org/10.1007/s10620-011-1875-8

[27] Shaker, H., Elsayed, H., Whittle, I., Hussein, S. and Shackcloth, M. (2010) The Influence of the "Golden 24-h Rule" on the Prognosis of Oesophageal Perforation in the Modern Era. European Journal of Cardio-Thoracic Surgery, 38, 216-222. https://doi.org/10.1016/j.ejcts.2010.01.030

[28] Qadeer, M., Dumont, J., Vargo, J. and López, R. (2007) Endoscopic Clips for Closing Esophageal Perforations: Case Report and Pooled Analysis. Gastrointestinal Endoscopy, 66, 605-611. https://doi.org/10.1016/j.gie.2007.03.1028

[29] Altorjay, A., Kiss, J., Voros, A. and Bohak, A. (1997) Nonoperative Management of Esophageal Perforations. Is It Justified? Annals of Surgery, 225, 415-421. 
https://doi.org/10.1097/00000658-199704000-00011

[30] Vogel, S., Rout, W., Martin, T. and Abbit, P. (2005) Esophageal Perforation in Adults: Aggressive, Conservative Treatment Lowers Morbidity and Mortality. Annals of Surgery, 241, 1016-1023. https://doi.org/10.1097/01.sla.0000164183.91898.74

[31] Port, J., Kent, M., Korst, R. and Bachetta, M. (2003) Thoracic Esophageal Perforations: A Decade of Experience. The Annals of Thoracic Surgery, 75, 1071-1074. https://doi.org/10.1016/S0003-4975(02)04650-7

[32] Eroglu, A., Turkylmaz, A., Ayden, Y., Yekeler, E. and Karaoglanoglu, N. (2009) Current Management of Esophageal Perforation: 20 Years Experience. Diseases of the Esophagus, 22, 374-380. https://doi.org/10.1111/j.1442-2050.2008.00918.x

[33] Holm, A.N., de la Mora Levy, J.G., Gostout, C.J., Topazian, M.D. and Baron, T.H. (2008) Self-Expanding Plastic Stents in Treatment of Benign Esophageal Conditions. Gastrointestinal Endoscopy, 67, 20-25. https://doi.org/10.1016/j.gie.2007.04.031

[34] Sharma, P. and Kozarek, R. (2009) Role of Esophageal Stents in Benign and Malignant Diseases. The American Journal of Gastroenterology, 105, 258-273.

https://doi.org/10.1038/ajg.2009.684

[35] Hasan, S., Jilaihawi, A. and Prakash, D. (2005) Conservative Management of Iatrogenic Esophageal Perforations-A Viable Option. European Journal of Cardio-Thoracic Surgery, 28, 7-10. https://doi.org/10.1016/j.ejcts.2005.03.006

[36] Johnsson, E., Lundell, L. and Liedman, B. (2005) Sealing of Esophageal Perforation or Ruptures with Expandable Metallic Stents: A Prospective Controlled Study on Treatment Efficacy and Limitations. Diseases of the Esophagus, 18, 262-266. https://doi.org/10.1111/j.1442-2050.2005.00476.x

[37] Fischer, A., Thomusch, O., Benz, S., von Dobschuetz, E., Baier, P. and Hopt, U.T. (2006) Nonoperative Treatment of 15 Benign Esophageal Perforations with Self-Expandable Covered Metal Stents. The Annals of Thoracic Surgery, 81, 467-472. https://doi.org/10.1016/j.athoracsur.2005.08.047

[38] White, R., Mungatana, C. and Topazian, M. (2003) Expandable Stents for Iatrogenic Perforation of Esophageal Malignancies. Journal of Gastrointestinal Surgery, 7, 715-719. https://doi.org/10.1016/S1091-255X(03)00064-7 